\section{Reflexões sobre a versão em Português da Classificação Internacional de Funcionalidade, Incapacidade e Saúde}

\author{
Comments on the Portuguese translation of the \\ International Classification of Functioning, \\ Disability and Health
}

1 Universidade de Brasília, Brasília, Brasil.

2 Instituto de Bioética,

Direitos Humanos e Gênero Brasília, Brasil.

3 International Poverty

Centre, Brasília, Brasil.

Correspondência

D. Diniz

Programa de Pós-graduação em Política Social,

Universidade de Brasília.

C. P. 8011, Brasília, DF

70673-970, Brasil.

anis@anis.org.br

\begin{abstract}
An analysis of the World Health Organization terminology for disability indicates the influence of the social model of disability in the International Classification of Functioning, Disability, and Health. This theoretical framework should guide any translations of the document. In Brazil, the document was translated as Classificação Internacional de Funcionalidade, Incapacidade e Saúde. We argue that more appropriate than translating disability as "incapacidade" and impairments as "deficiências" would be to use the term "deficiência" for disability and "lesão" for impairment. Considering the normative impact of a WHO document for social policy and international research, the translation should accurately reflect the concepts and their theoretical basis.
\end{abstract}

Terminology; Diseases Classification; Public Policies
Debora Diniz 1,2

Marcelo Medeiros 2,3

Flávia Squinca 2

A publicação da International Classification of Functioning, Disability and Health (ICF) pela Organização Mundial da Saúde (OMS), em 2001, é considerada um marco no debate sobre deficiência 1. O documento é uma revisão da International Classification of Impairments, Disabilities, and Handicaps (ICIDH), primeira tentativa da OMS de organizar uma linguagem universal sobre lesões e deficiências, publicada em $1980^{2}$. Dentre as várias modificações propostas pela ICF, uma das mais desafiantes foi o novo significado do termo "deficiência”. De uma categoria estritamente biomédica na ICIDH, deficiência assumiu um caráter também sociológico e político na ICF.

Essa redefinição da deficiência foi resultado de um extenso debate acadêmico já anterior à elaboração da ICIDH, porém intensificado com a publicação do documento. De acordo com a ICIDH, haveria uma relação de causalidade entre impairments (perdas ou anormalidades corporais), disabilities (restrições de habilidade provocadas por lesões) e handicaps (desvantagens resultantes de impairments ou disabilities) ${ }^{2}$. Um corpo com lesões experimentaria restrições de habilidades, o que levaria a pessoa a situações de desvantagem social. A desvantagem seria resultado das lesões, por isso a importância de conhecer, curar ou reabilitar os corpos anormais. 
A linguagem proposta pela ICIDH foi alvo de severas críticas do então emergente campo dos estudos sobre deficiência (disability studies). Em resposta à hegemonia biomédica sobre o tema, os estudos sobre deficiência surgiram como uma especialidade das humanidades em saúde, cujo compromisso teórico era demonstrar que a experiência da desigualdade pela deficiência resultava mais de estruturas sociais poucos sensíveis à diversidade que de um corpo com lesões. O modelo social da deficiência - principal marco teórico dos estudos sobre deficiência - subverteu a lógica da causalidade proposta pela ICIDH: não eram as lesões a principal causa das desvantagens, mas sim a opressão social aos deficientes.

Foram vinte anos de debates entre o modelo biomédico e o modelo social da deficiência, e o principal resultado foi a revisão do sistema classificatório da OMS. Não é por acaso que a OMS faz referências aos dois modelos na ICF e afirma ser a nova linguagem uma combinação de ambos para a instauração de um terceiro, o modelo biopsicossocial. Mas como qualquer campo emergente, um dos temas de maior disputa é sobre como descrever os fenômenos e as novas especialidades. Ciente da importância da linguagem para o sucesso da ICF como um vocabulário universal, a OMS propôs uma redefinição ampla da deficiência, ao ponto de afirmar que "há uma ampla incompreensão de que a ICF seja somente sobre pessoas com deficiência; na verdade, é sobre todas as pessoas" 1 (p. 8).

A afirmação de que a ICF é um documento de caráter universal pode ser entendida de duas maneiras. A primeira como um reconhecimento da força política do modelo social da deficiência para a revisão do documento: de uma classificação de corpos com lesões (ICIDH) para uma avaliação complexa da relação indivíduo e sociedade (ICF). Uma pessoa com deficiência não é simplesmente um corpo com lesões, mas uma pessoa com lesões vivendo em um ambiente que oprime e segrega o deficiente. A segunda maneira de compreender a afirmação universalista da ICF é também resultado do modelo social: a deficiência não é uma tragédia individual ou a expressão de uma alteridade distante, mas uma condição de existência para quem experimentar os benefícios do progresso biotecnológico e envelhecer. Velhice e deficiência são conceitos aproximados pela ICF e pela nova geração de teóricos da deficiência 3 .

Mas nesse processo de revisão da ICIDH para ICF uma das questões mais sensíveis foi sobre como descrever o fenômeno da deficiência. A ICIDH utilizava os termos impairments, disabilities e handicaps. Como impairments e disabilities foram variáveis descritas em termos biomédicos na ICIDH, isto é, com base em estatísticas de normalidade e em curvas de variação sobre os padrões corporais, acreditava-se que a controvérsia sobre os dois conceitos seria atenuada, o que de fato não ocorreu. A demanda do modelo social da deficiência era por descrever as lesões como uma variável neutra da diversidade corporal humana, entendendo o corpo como um conceito representativo da biologia humana. O sistema proposto pela ICIDH classificava a diversidade corporal como conseqüência de doenças ou anormalidades, além de considerar que as desvantagens eram causadas pela incapacidade do indivíduo com lesões de se adaptar à vida social.

Para o modelo social, a ICIDH retirava a força política do conceito de deficiência, pois o fundamentava em termos estritamente biológicos: era a natureza quem determinava a desvantagem, e não os sistemas sociais ou econômicos. Argumentou-se que o conceito de handicap resgataria o que se chamou de "componente extrínseco" da deficiência, ou seja, suas variáveis não biomédicas. A tese do "componente extrínseco" da deficiência esteve reservada a um apêndice pouquíssimo explorado no modelo tripartido da ICIDH, a tal ponto que alguns autores consideraram tal componente um conceito sem significado teórico para as propostas classificatórias do documento ${ }^{4}$.

A revisão da ICF procurou resolver essa controvérsia, incorporando as principais críticas do modelo social. Pelo novo vocabulário, disability é um conceito guarda-chuva que engloba o corpo com lesões, limitações de atividades ou restrições de participação 1 . Ou seja, disability não se resume a impairment, pois é o resultado negativo da interação de um corpo com lesões em ambientes sociais pouco sensíveis à diversidade corporal das pessoas. O objetivo dessa redefinição de disability foi incorporar a crítica dos teóricos do modelo social: deficiência é uma experiência sociológica e política e não apenas o resultado de um diagnóstico biomédico sobre corpos anômalos. Foi também com esse espírito que o conceito de handicap foi definitivamente abandonado, em especial pela etimologia que remetia os deficientes a pedintes ("chapéu na mão").

A OMS reconheceu o desafio de redescrição conceitual do campo e o Anexo 1 antecipa a dificuldade de tradução que o novo documento provocará: "ICF, como uma classificação escrita, será traduzida para muitos idiomas. Além de uma compreensão comum dos conceitos, é importante que um acordo seja alcançado sobre o termo que melhor reflete o conteúdo em cada idioma. Há muitas alternativas e as decisões devem ser tomadas baseadas na acuidade, aceitabilidade e utilidade em geral" 1 (p. 188). A Língua Portugue- 
sa não é um dos idiomas oficiais da OMS, o que torna a tradução local uma iniciativa solidária de pesquisadores cientes da importância da ampla divulgação do documento. O sucesso de uma tradução não deve ser medido apenas pela escolha adequada dos termos correspondentes em cada idioma, mas também pela fidelidade ao debate teórico e político que fundamentou os termos originais.

No Brasil, a ICF foi traduzida pelo Centro Colaborador da OMS para a Classificação de Doenças em Português, da Universidade de São Paulo, e o título traduzido foi: Classificação Internacional de Funcionalidade, Incapacidade e Saúde 5.0 termo escolhido para traduzir disability foi "incapacidade". Talvez os tradutores tenham apostado na aceitabilidade do conceito de "incapacidade" no léxico ativo do idioma para representar o espírito guarda-chuva sugerido pela ICF para disability. Outra possível explicação é que a entrada em cena de um novo conceito facilitaria o trabalho de aceitação do vocabulário proposto pela ICF. O fato é que não há uma explicação dos tradutores para essa escolha, assim como foram apresentadas justificativas para outras traduções também desafiantes, tais como construct ou attitudinal.

No entanto, "incapacidade" ignora trinta anos de debate acadêmico e político internacional, em especial as contribuições do modelo social para a revisão da ICF. A única tradução possível para disability - acurada e aceitável para o marco teórico que inspirou a revisão da ICF - é deficiência. Disability e deficiência são conceitos carregados de conteúdo normativo para o universo biomédico, e essa não é uma particularidade da Língua Portuguesa. Mas a escolha de disability e não outra categoria foi exatamente para provocar a tradição biomédica de dois séculos que compreende deficiência como algo fora da norma 6 . Havia um objetivo político e moral por trás da escolha de disability: desestabilizar a hegemonia biomédica no campo.
A tradução brasileira ignorou esse debate, ou ao menos não o explicitou por ocasião da publicação da versão traduzida, o que torna a tradução pouco coerente com os fundamentos teóricos do documento. Outro indício desse fenômeno foi a tradução de impairments por deficiências. Para o modelo social, impairments representam as lesões, isto é, as variações corporais, ao passo que disability é o que expressa a interação do corpo com a sociedade. Esse é um tema onde as escolhas conceituais importam e não apenas pelo caráter em disputa do campo já mencionado. Há uma tentativa de redescrição acadêmica, política e moral da deficiência, um esforço conjunto de diferentes saberes por reconhecer a deficiência como uma expressão da desigualdade social. O neologismo disablism, cuja tradução seria a ideologia que oprime o corpo deficiente, isto é, a “a opressão pela deficiência”, resume esse fenômeno: há uma ideologia que oprime e segrega o corpo com lesões 7 .

A ambição da ICF é por se constituir em uma linguagem universal para políticas públicas, pesquisas científicas e iniciativas internacionais comparativas. Por isso, o lapso de tradução da versão brasileira necessita ser revisto para a legitimidade e acuidade de um campo emergente no país: são pessoas com deficiência (people with disability) ou deficientes (disabled ou disabled people) e não pessoas com incapacidades ou incapacitados. Deficiência não foi uma categoria redescrita apenas no universo biomédico, mas principalmente a partir da contribuição de diferentes saberes, em especial o modelo social da deficiência e dos movimentos sociais de deficientes nas últimas três décadas. Nesse sentido, nossa proposta é que seja resgatado o caráter original do debate teórico que inspirou a ICF, assumindo que a categoria-chave do documento é deficiência é não incapacidade. 


\section{Resumo}

A análise da terminologia adotada pela Organização Mundial da Saúde para deficiência mostra que a International Classification of Functioning, Disability and Health foi fortemente influenciada pelo modelo social da deficiência, um campo de pesquisas sobre deficiência das humanidades em saúde. As traduções do documento devem contemplar esse marco teórico nas escolhas terminológicas em cada idioma. No Brasil, o documento foi traduzido como Classificação Internacional de Funcionalidade, Incapacidade e Saúde. Julgamos que mais adequado que traduzir disability por "incapacidade" e impairments por "deficiências" seria usar o termo "deficiência" para disability e "lesão" para impairment. Dado o marco normativo do documento para as políticas públicas e pesquisas científicas é preciso garantir a acuidade e legitimidade dos conceitos e seus fundamentos teóricos.

Terminologia; Classificação de Doenças; Políticas Públicas

\section{Colaboradores}

Os três autores foram responsáveis pela redação da nota de pesquisa.

\section{Referências}

1. World Health Organization. International classification of functioning, disability and health. Geneva: World Health Organization; 2001.

2. World Health Organization. International classification of impairments, disabilities, and handicaps. Geneva: World Health Organization; 1980.

3. Wendell S. The rejected body: feminist philosophical reflections on disability. New York: Routledge 1996.

4. Edwards SD. Dismanteling the disability/handicap distinction. J Med Philos 1997; 22:589-606.
5. Centro Colaborador da Organização Mundial da Saúde para a Família de Classificações Internacionais, organizador. CIF: Classificação Internacional de Funcionalidade, Incapacidade e Saúde. São Paulo: Editora da Universidade de São Paulo; 2003.

6. Stiker HJA. History of disability. Michigan: The University of Michigan Press; 1997.

7. Albrecht G, Seelman K, Bury M. Handbook of disability studies. London: Sage Publications; 2001.

Recebido em 27/Fev/2007

Versão final reapresentada em 21/Jun/2007

Aprovado em 29/Jun/2007 